\title{
Texture Features to Evaluate Biometric Verification System using Handvein and Palmprint
}

\author{
Shreyas Rangappa \\ Research Scholar \\ SJBIT,Banglore
}

\author{
Naveena C. \\ Professor \\ SJBIT, Banglore
}

\author{
H. K. Chethan \\ Professor \\ MIT, Mysuru
}

\author{
G. Hemantha Kumar \\ Professor \\ University of Mysore
}

\begin{abstract}
In recent years multimodal biometrics plays vital role in real life scenarios. We have proposed evaluated a biometric verification system of universal acceptable hand based modalities. We have used the Dorsal Handvein and Palmprint traits that are recently emerging traits in the multimodal biometrics field. We used the well know texture methods like LBP, LPQ and Gabor filter to extract texture features on Handvein and Palm print databases. We compare results of texture methods individually and also we worked on combinations of all the features on both the modalities and which modality performs better on texture descriptors. We have shown results using GAR (Genuine Acceptance Rate) v/s FAR (False Acceptance Rate) with the threshold benchmark values of $\operatorname{FAR}(0.01 \%, 0.1 \%, 1 \%)$ to illustrate the performance of verification rate. At the last we have tested with Multi-algorithmic system to evaluate robustness of our system. From our experimental results, it is clearly evident that the $\mathrm{LPQ}+$ Gabor combination texture feature is more suitable for both modalities.
\end{abstract}

\section{Keywords}

LBPV, Multi-algorithm, LPQ, Palmprint, Handvein

\section{INTRODUCTION}

Now a days most of the applications are based on secure accessing of openings to buildings, banks, computers, laptops, mobiles and ATMs. Usually identifying an individual is based on user name and password systems or smartcard and password systems. The process of granting the permission to access or denying the access to a network resource consists of two steps. The first step is authentication which ensures that whether a user is he or she who claims to be. The second step is authorization which allows the user to access the various resources of a network.

Confidential transaction needs highly secure biometric systems to avoid fraudulent actions. Traditionally, individual's identity is determined by What You Have (Tokens), What You Know (Passwords) and What You Are (Biometric trait). Conventional methods are fail to meet the desirable requirements. Biometric identifiers are more reliable than token based and knowledge based identification systems, because they rely on substitute representations. The biometric authentication is done either by identification or verification. Biometric Identification systems are described as a 1-to-n matching system that reads a sample and compares the sample against every template already in the database and the authentication is done to access the system [16]. Biometric verification refers to verify an individual by matching the new biometric sample with the specific biometric already stored in the database, it is also called as 1-to-1 matching system, the cost of verification is less compared to identification. In this work, we have used two recently developing modalities namely handvein and Palm
Print. Handvein is one of the safest biometric and the patterns are underlying in the skin, so the falsification is difficult. This modality is network of blood vessels, stable, contactless image acquisition, difficult to forge, hard to damage and even the twins have different handveins. Hand veins are not used widely, due to less availability of the databases and the cost of acquisition device is high. On the other hand the Palmprint recognition has drawn a lot of attention during the recent years. Many algorithms have been proposed for palmprint recognition in the past, majority of them being based on features extracted from the transform domain. Many of these transform domain features are not translation or rotation invariant, and therefore a great deal of preprocessing is needed to align the images.

In general every biometric system works in this flow, Sensor module: a quality checking module which acquires the biometric data of an individual. Preprocessing of raw data is necessary. Feature extraction module: captures biometric data and processes to extract salient features. Matching module: also called as decision making module that measures the similarity of features during recognition with a reference template by applying various methods like probabilistic measures, neural networks, etc. Decision module maintains the expressive templates for enrolled users as a reference for future comparison in a central database. Identified features are compared by the system with the stored reference to establish whether claimed identity is accepted or rejected [20]. Performance parameters used for verification of biometric are Equal Error Rate (EER): Predetermination of threshold values for its false acceptance rate and its false rejection rate, FAR $=$ FRR, also called as crossover error rate (CER). False Acceptance Rate (FAR): A statistic used to measure biometric performance where invalid inputs are incorrectly matched to another biometric subject when operating in the verification task. Genuine Acceptance Rate (GAR): is an overall accuracy measurement of a biometric system. It is calculated by the formula: $\mathrm{GAR}=1-\mathrm{F}$ RR. Receiver Operating Characteristics (ROC): ROC curve is plotted to analyze the performance of the system graphically, the threshold is computed and performance metrics used in plotting ROC graph are GAR and FAR [15].

Handvein and Palm Print biometrics have many advantages that makes it preferable in many biometric applications such as acceptability, availability of strong feature extraction algorithms, easy for image acquisition etc. On the other hand, even though there exists several studies in the literature that have showed on applying particular texture based algorithms on these two modalities, there are no evidences of solitary work that reports fusion method for handvein and Palmprint. In order to evaluate the texture feature extraction algorithms of these two modalities in real time scenarios, performance analysis needs to be carried out. In this work, we have evaluated the performance of texture 
feature extraction algorithms and Multi-algorthmic models for Handvein and Palmprint. The organization of the paper is as follows: Section 2 gives review of literature related to Handvein and Palmprint biometric systems. Section 3 describe the proposed method. Section 4 discusses experimental results. Conclusion and future work are drawn in Section 5.

\section{REVIEW OF LITERATURE}

Ali Younesi et al. [1] proposed a palmprint recognition method for an systematic personal identification system. ROI of palmprint is obtained and then acquired ROI is given to Gabor filter with arbitrary orientations and wavelengths. Binarized statistical image features are used to extract texture information by outputs of Gabor filter and then histogram features are obtained from binarized statistical image features code and is passed through $\mathrm{KNN}$ classifiers to execute the personal identification. Shrevin Minaee et al. [2] proposed a palmprint recognition algorithm model by employing convolution scattering network. Scattering network is capable of capturing almost identical features to Scale Invariant Feature Transform(SIFT) and alternative descriptors from the first layer and high frequency content from the elevated layers. Once features are extracted Principal Component Analysis is applied to decrease their dimensionality. For recognition they have employed multiclass SVM and nearest neighbor classifiers. Haipeng Chen et al. [3] An hybrid hierarchical multispectral palmprint recognition model by developing block dominant orientation code to capture texture information successfully in spatial domain. Hierarchical matching and feature level fusion method based on block selection is used to increase the accuracy and security of the system. Saiyed Umer et al. [4] proposed a palmprint recognition model which comprises of pre-processing, feature extraction and classification. For an effective pre-processing of ROI of palm image thresholding and mathematical morphological method is obtained. To extract features patch based filter responses is adopted and gives effective results than other methods for analyzing textural information. Jan Svoboda et al. [5] proposed a palmprint recognition using deep convolution neural networks (CNN). For training the network they have employed new d-prime loss index which is capable of increasing the separation of genuine and impostor score distributions. Lunke Fei et al. [6] proposed a palmprint recognition method based on discriminative neighboring direction indictor that will represent the orientation feature of the palmprint. Almost all dominant orientations feature of palmprint is represent by discriminative neighboring direction indicator and the most important is it also represents orientation feature of various points considering double dominant orientations. Main advantage is discriminative orientation indicator is strong to rotation and noise. Gen Li et al. [7] proposed a palmprint recognition method which is built on unique local descriptor called Local Micro-structure Tetra Pattern (LMTrP). The proposed descriptors are incorporated with line-shaped based filters. Main advantage is that it utilization of local descriptors, thickness and direction that is capable of increasing the performance. Yue-Tong Luo et al. [8] proposed a palmprint recognition method which uses local line directional patterns (LLDP) and works on local line-geometry space which is a new feature that is define from Local binary patterns like descriptors. The proposed feature LLDP which make use of directional indexing will provide better results compared to other methods such as Gabor based implementation of LLDP.
Di Huang et al. [9] proposed a handvein recognition model employing LBP (Local Binary Pattern) and BC (Binary Coding) to extract either the texture or shape features of hand vein modality and the features are represented using the Graphical notation. The vein shape features are extracted by minutiae features and by a BC (binary coding) to analyze skeletal image, the image is intensified using the Gaussian filter. LBP, BC are fused to arrive at a final decision. The number of images are increased in the gallery and accuracy at higher level is achieved. Jun Wang et al. [10] proposed hand vein recognition system, they have used a relatively cheap charge coupled device (CCD) camera lens of infrared light to record the underlying hand vein images at lower resolution, Segmentation is the challenging task and vital aspect in biometric authentication, where the background image has to be separated and the ROI (Region of Interest) should be extracted. The Gaussian low pass filter is applied on original images to remove the high spatial frequency and the binary images obtained from Niblack algorithm are subjected to sobel operator to emphasize regions, The boundary is determined to extract the ROI of the image, PCA, LDA and Gabor algorithms are used for classification. The structure of Hand vein is a time invariant property and detected only live. Due to uneven illumination and difficulty in extracting ROI (region of interest), preprocessing is a challenging task and obtaining feature points in Handvein Biometric modality. Inshirah Rossan and Maleika HeenayeMamode Khan [11] proposed preprocessing techniques for hand vein images. The contrast of the original image is enhanced by histogram equalization, The Contrast limited adaptive histogram equalization (CLAHE) is employed for preprocessing followed by mean-c, median-c and wellner's adaptive threshold. Threshold is done to separate the background from foreground image, FAR (False Acceptance Rate) and FRR (False Reject Rate) are used as performance measures. Yun peng $\mathrm{Hu}$ et al. [12] proposed a hand vein recognition model. The Geometric operation, rotation when subjected on hand vein images leads to degradation of system performance. These drawbacks have been addressed in their work, the interconnection points in the hand vein images like intersection point and terminating end points are used as features. False acceptance rate is one of the performance measure which cannot be ignored while considering the biometric authentication system's accuracy. Ahmed M. Badawi [13] proposed a hand vein verification system, the shape and size of the hand vein modality are the features to be extracted. The vein tree is different from each individual and the vein pattern of both the hands of an individual is also different, Gaussian filter is used in smoothening and the edges are also retained to analyze statistical performance measures. ROC curve is plotted to analyze the performance of the system graphically. Aditya Nigam et al. [14] proposed a Palmprint based system. The ROI regions, central knuckle line and central knuckle point is extracted by Gabor filter, the image samples are enhanced using CLAHE (Central Limited Adaptive Histogram Equalization) for better discrimination, the images are binarized and edges are detected using the canny edge detection method, ROC curve is plotted and EER(Equal Error Rate) is reported.

\section{PROPOSED METHOD}

In this work, we have used the hand based traits for our experimentation, from the literature these traits are highly discriminate by the texture. Nevertheless, we have applied texture descriptors such as Gabor filter, LPQ and LBPV. We 
have tested multi-algorithmic models to test which combination of texture method is robust.

\subsection{Gabor FilterReponses}

Gabor filter is a local texture descriptor named after Dennis Gabor. Gabor wavelet represents spatial orientation and frequency characteristics and similar to human visual system, similar to windowed Fourier transform [16]. It is a multi-resolution filter used in many applications. $2 \mathrm{D}$ wavelet decomposition with Gabor filter is applied to extract local features. The 2D gabor filter is modulated by gaussian kernel and complex sinusoidal function, on applying gabor filter to obtain real and imaginary features with orientation $\theta$ and frequency $f$ is given by

$$
f\left(x, y, f, \theta, \sigma_{x}, \sigma_{y}\right)=\frac{1}{2 \pi \sigma_{x}, \sigma_{y}} \exp \left\{-\frac{x^{2}+y^{2}}{2 \sigma^{2}}+f(x \cos \theta+y \sin \theta)\right.
$$

$\sigma_{x}$ and $\sigma_{y}$ are standard deviations along $\mathrm{x}$ and $\mathrm{y}$ directions.

\subsection{LPQ (Local Phase Quantization)}

The Local Phase Quantization (LPQ) is originally proposed by [17] for texture based Pattern classification, which is blur insensitive and rotation invariant. This texture feature is robust compared to Gabor and LBP [17] from this evident we used this to verification of Palmprint and Handvein. LPQ is a fourier transform quantizer, Fourier domain using the convolution theorem is given by, $\mathrm{G}(\mathrm{u})=\mathrm{F}(\mathrm{u}) \cdot \mathrm{H}(\mathrm{u})$, where $\mathrm{G}, \mathrm{F}$, and $\mathrm{H}$ are discrete Fourier transforms. $\mathrm{H}(\mathrm{u})$ is the point spread function of the blur image and $F(u)$ is the original image. The magnitude and phase of the image can be separated by,

$$
|G(u)|=|F(u)| *|H(u)|
$$

The Fourier transform is a two valued function and and $\mathrm{H}$ must equal 0 or $\Pi$. The phase is computed by imaginary and real points of the feature vector $f_{x}$.

$$
f_{x}=\left[\operatorname{Re} f_{x}^{c}, I_{m} f_{x}^{c}\right] \text { where }\left[F\left(u_{1}, x\right), F\left(u_{2}, x\right), F\left(u_{3}, x\right), F\left(u_{4}, x\right)\right]_{(3)}
$$

and the frequency vectors are given by, $u_{1}=[a, 0]^{T}, u_{2}=$ $[0, a]^{T}, u_{3}=[a, a]^{T}, u_{4}=[a, a]^{T}$.

\subsection{LBPV}

Local Binary Pattern and Its Variant (LBPV) The original LBP operator, introduced by Ojala et al. [19] in 1996. LBP is a local texture operator proposed by [16], which has a low computational complexity and a low sensitivity to changes in illumination. The LBP have been tested in several applications, for example, in texture classification [16], face recognition [21], smart gun [22], fingerprint identification [23], and automated cell phenotype image classification [24]. LBPV proposes an alternative hybrid scheme; globally rotation invariant matching with locally variant LBP texture features. It is a simplified but efficient joint LBP and contrast distribution method. LBPp,r /VARp,r is powerful because it exploits the complementary information of spatial pattern and local contrast. Threshold values are used to quantize the VAR of the test images computed to partition the total distribution into $\mathrm{N}$ bins with an equal number of entries. A central pixel is selected in the input image and the binary code is calculated by thresholding the neighborhood over the center pixel. If the center pixel's value is greater than the neighboring value, then the binary value is set to ' 1 ' else the binary value is set to ' 0 '. If the value of the neighboring pixels does not change, then it is featureless. If there is at most two transitions in the cell then it is called uniform pattern, else if it contains more than two transitions it is said to be non-uniform pattern. The basic 8-bit version of the LBP operator considers only eight neighbors which is of 256 dimensional vector, which is used as a texture descriptor. Histogram of all the independent cells is computed and the feature vectors are normalized. LBPV is a rotation invariant descriptor that descriptors the local contrast information, higher variance has more discriminative texture features. Guo et al. [18] proposed the calculation adaptive weight in histogram of LBP code, the LBPV histogram is computed as,

$$
\operatorname{LBPV}_{p, r}(K)=\sum_{i=1}^{n} \sum_{j=1}^{m} W\left[\left(L B P_{p, r}(i, j), K \in(0, K)\right)\right] \text { (4) }
$$

where $\mathrm{K}$ indicates the maximal value of LBP codes.

\section{EXPERIMENTAL RESULTS}

In this section we described how our system is good with different texture methods and also we tested combination of multi-algorithms on proposed hand modalities. In this work we considered two hand modalities such as Handvein from Cluj University database and Palmprint from PolyU Palmprint database. In Handvein modality we have considered 100 users such that each user has 5 samples of Handvein image, in that 3 samples for training and 2 for testing. In Palmprint database 100 users, each user have 10 samples and out of 10 samples we have chosen 6 samples for training and 4 samples for testing.

Basically texture methods are separated into structural and statistical. In this work we have used recent state-of art methods namely Gabor, LPQ, LBPV. From Gabor 5076 features are extracted and from LPQ and LBPV 256 features are extracted respectively. For evaluation of each texture method we calculated GAR (Genuine Acceptance Rate) and FAR (False Acceptance Rate) at $0.01 \%, 0.1 \%$ and $1 \%$ threshold ratings.

The Performance of texture methods on Handvein Modality is shown in Table.1 and Palmprint modality is in Table.2.

Table 1. Performance of different texture feature extraction algorithms for Handvein

\begin{tabular}{|l|l|l|l|}
\hline \multirow{2}{*}{ FAR\% } & \multicolumn{2}{|l|}{ GAR \% } \\
\cline { 2 - 4 } & LBPV & Gabor & LPQ \\
\hline 0.01 & 7 & 35.2 & 30.7 \\
\hline 0.1 & 18 & 57.3 & 40 \\
\hline 1 & 39 & 78.6 & 56.3 \\
\hline
\end{tabular}

From the Table.1. We easily conclude that Gabor method is outperformed compared to other texture methods with GAR $\%$ and FAR $\%$ of $1 \%$. So Handvein Modality is gives good performance for Gabor compared to other texture methods.

Table 2. Performance of Multi-algorithmic of texture feature for Handvein

\begin{tabular}{|l|l|l|l|}
\hline \multirow{2}{*}{ FAR\% } & \multicolumn{2}{|l|}{ GAR \% } \\
\cline { 2 - 4 } & LBPV & LPQ & LBPV \\
& + & + & + \\
& Gabor & Gabor & LPQ \\
\hline
\end{tabular}




\begin{tabular}{|l|l|l|l|}
\hline 0.01 & 10 & 38.2 & 32.5 \\
\hline 0.1 & 21 & 58.6 & 42.6 \\
\hline 1 & 43 & 80.7 & 58.3 \\
\hline
\end{tabular}

\section{REFERENCES}

[1] Ali Younesi, Mehdi Chehel Amirani,: Gabor Filter and Texture Based Features for Palmprint Recognition, Iternational Conference on Computational Science, ICCS Procedia Computer Science, 2017.

Table 3. Performance of different texture feature extraction algorithms for Palmprint

\begin{tabular}{|l|l|l|l|}
\hline \multirow{2}{*}{ FAR\% } & \multicolumn{2}{|l|}{ GAR \% } \\
\cline { 2 - 4 } & LBPV & Gabor & LPQ \\
\hline 0.01 & 16 & 85.4 & 86.7 \\
\hline 0.1 & 22 & 88 & 90.3 \\
\hline 1 & 40 & 91.7 & 93.4 \\
\hline
\end{tabular}

Table 4. Performance of Multi-algorithmic of texture feature for Palmprint

\begin{tabular}{|l|l|l|l|}
\hline \multirow{4}{*}{ FAR\% } & \multicolumn{2}{|l|}{ GAR \% } \\
\cline { 2 - 4 } & $\begin{array}{l}\text { LBPV } \\
+\end{array}$ & $\begin{array}{l}\text { LPQ } \\
+\end{array}$ & $\begin{array}{l}\text { LBPV } \\
+ \\
\end{array}$ \\
& Gabor & Gabor & LPQ \\
\hline 0.01 & 18 & 88.2 & 72.5 \\
\hline 0.1 & 25 & 89.8 & 68.6 \\
\hline 1 & 46 & 94.7 & 78.3 \\
\hline
\end{tabular}

When we look into Palmprint modality with all the texture methods, it fail to give good performance for LBPV and Gabor compared LPQ. LPQ give good result with GAR\% 93.4 and highest FAR $\%$ of $1 \%$. From the Table 1. And Table 3. We can easily conclude that all types of texture methods are not suitable for all the modalities in biometrics. So, that we proposed combination of texture methods to improve the results. Combination of two texture method means Multi-Algorithmic model. When we using single texture methods we have achieved good results but compared to single texture method combination of two texture methods gives good results. In this way we have tested Multi-Algorithmic model on Hanvein and Pamlprint modalities and show the results in Table 2. and Table 4 . respectively. Combination of two texture methods is outperformed compared to individual texture methods.

\section{CONCLUSION AND FUTURE WORK}

In this work we have proposed Texture based methods to evaluate Handvein and Palmprint biometrics. In the prosed method describes how we used Gabor filter, LBP, LPQ texture methods and also we used Mutli-algoorithms means to test robustness of texture methods. In the experimental results shows that how we get better performance on different texture methods with Handvein and Palmprint traits and also we have shown results of Multi-algorihtmic models. Overall evaluation LPQ+Gabor gives good performance in Multi-algorithmic models and Gabor method gives good performance for Handvein and LPQ gives better results for Palmprint. In future work we are planning to explore these texture methods with feature fusion, score level fusion.

[2] Shrevin Minaee, Yao Wang,: Palmprint Recognition using Deep Scattering Convolution Network, 2016.

[3] Haipeng Chen,: An Efficient Palmprint Recognition method Based on Block Dominant Orientation Code, Elsevier 2015.

[4] Saiyed Umer, Bibhas Chandra Dhara, Bhabatosh Chanda,: A Novel Palmprint Recognition System using Patch Based Filter Response, IEEE International Conference on Identity, Security and Behavior Analysis(ISAB), 2017.

[5] Jan Svoboda, Jonathan Masci, Michael M. Bronstein,: Palmprint Recognition via discriminative index learning, International Conference on Pattern Recognition (ICPR), 2016.

[6] Lunke Fei, Bob Zhang, Yong $\mathrm{Xu}$, Liping Yan,: Palmprint Recognition Using Neighboring Direction Indicator, IEEE Transactions on Human-Machine Systems, volume 46, 2016.

[7] Gen Li, Jaihie Kim,: Palmprint recognition with Local Micro-structure Tetra Pattern, Elsevier, Pattern Recognition 61, 2017, 29-46.

[8] Yue-Tong Luo, Lan-Ying Zhao, Bob Zhang, Wei Jia, Feng Xue, Jing-Ting Lu, Yi-Hai Zhu, Bing-Qing Xu,: Local Line Directional Pattern for palmprint recognition, Elsevier, Pattern Recognition 50, 2016, 26-44.

[9] Huang, D., Zhu, X., Wang, Y., Zhang, D.: Dorsal hand vein recognition via hierarchical combination of texture and shape clues. Neurocomputing 214(C), 815-828 (2016). ISSN 0925-2312

[10] Wang, J., Wang, G., Li, M., Wenkai, D.: Hand vein recognition based on PCET. Optik 127, 7663-7669 (2016)

[11] Rossan, I., Khan, M.H.M.: Impact of changing parameters when preprocessing dorsal hand vein pattern. Procedia Comput. Sci. 32, 513-520 (2014)

[12] Hu, Y.-P., Wang, Z.-Y., Yang, X.-P., Xue, Y.-M.: Hand vein recognition based on the connection lines of reference point and feature point. Infrared Phys. Technol. 62, 110-114 (2014)

[13] Badawi, A.M.: Hand vein biometric verification prototype: a testing performance and patterns similarity. IPCV 14, 3-9 (2006)

[14] Nigam, A., Tiwari, K., Gupta, P.: Multiple texture information fusion for finger knuckleprint authentication system. Neurocomputing 188, 190-205 (2016).

[15] Raghavendra, R., Imran, M., Rao, A., Hemantha Kumar, G.: Multimodal biometrics: analysis of handvein and palmprint combination used for person verification. In: 2010 3rd International Conference on Emerging Trends in Engineering and Technology (ICETET). IEEE (2010). 
[16] Ojala, T., Pietik"ainen, M., Harwood, D.: A comparative study of texture measures with classification based on feature distributions. Pattern Recogn. 29(1), 51-59 (1996)

[17] Ojansivu, V., Heikkila, J.: Proceedings of the 3rd International Conference on Image and Signal Processing, ICISP 2008, pp. 236-243 (2008)

[18] Guo, Z., Zhang, L., Zhang, D.: A completed modeling of local binary pattern operator for texture classification. IEEE Trans. Image Process. 19, 16571663 (2010).

[19] Lumini, A., Nann, L.: Overview of the combination of biometric matchers. Inf. Fusion 33, 71-85 (2017). Original Research Article.

[20] Khandelwal, C.S., Maheshewari, R., Shinde, U.B.: Review paper on applications of principal component analysis in multimodal biometrics system original research article. Procedia Comput. Sci. 92, 481-486 (2016).

[21] Nanni L, Lumini A. RegionBoost learning for 2D + 3D based face recognition. Pattern Recogn Lett 2007;28(15):2063-70.

[22] Shang X, Veldhuis R. Local absolute binary patterns as image preprocessing for grip-pattern recognition in smart gun. In: Proceedings of the first IEEE international conference on biometrics: theory, applications, and systems; 2007. p.1-6.

[23] Nanni L, Lumini A. Local binary patterns for a hybrid fingerprint matcher. Pattern Recogn 2008;11:3461-6.

[24] Nanni L, Lumini A. A reliable method for cell phenotype image classification. Artif Intell Med 2008;43(2):87-97. 